\title{
Fast computer-generated hologram computation using rendered depth map image
}

Seyedmahdi Kazempourradi, Erdem Ulusoy, Hakan Urey

Seyedmahdi Kazempourradi, Erdem Ulusoy, Hakan Urey, "Fast computergenerated hologram computation using rendered depth map image," Proc. SPIE 10127, Practical Holography XXXI: Materials and Applications, 101270Q (6 April 2017); doi: 10.1117/12.2252374

SPIE. Event: SPIE OPTO, 2017, San Francisco, California, United States 


\title{
Fast computer-generated hologram computation using rendered depth map image
}

\author{
Seyedmahdi Kazempourradi ${ }^{a}$, Erdem Ulusoy ${ }^{\mathrm{a}}$, and Hakan Urey ${ }^{\mathrm{a}}$ \\ ${ }^{a}$ Koc University, Electrical Engineering Department, Optical Microsystems Laboratory, \\ Istanbul, Turkey
}

\begin{abstract}
We propose a method for computing realistic computer-generated holograms (CGHs) of three-dimensional (3D) objects, where we benefit from well-established graphical processing units (GPUs) and computer graphics techniques to handle occlusion, shading and parallax effects. The graphics render provides a 2D perspective image including occlusion and shading effects. We also extract the depth map data of the scene. The intensity values and 3D positions of object points are extracted by combining the rendered intensity image and the depth map (Z-buffer) image. We divide the depth range into several planes and quantize the depth value of 3D image points to the nearest plane. In the CGH computation part, we perform proper Fresnel transformations of these planar objects and sum them up to create the hologram corresponding to the particular viewpoint. We then repeat the entire procedure for all possible viewpoints and cover the hologram area. The experimental results show that the technique is capable of performing high quality reconstructions in a fast manner.
\end{abstract}

Keywords: Holography, Computer-Generated Hologram, Computer Graphics, GPU

\section{INTRODUCTION}

Holographic displays can reproduce a three-dimensional (3D) scene with all natural depth cues. Spatial light modulators (SLMs) combined with computer-generated holograms (CGHs) synthesize wavefronts that are indistinguishable from those emanated by physical objects. CGH computation techniques can roughly be classified into two main groups: wave-propagation methods ${ }^{1,2}$ and ray-tracing-based ${ }^{3}$ methods.

In wave-propagation methods, objects are represented by a set of self-emitting point sources (the point cloud model) or polygons (mesh model). The optical wave field of a 3D scene is reconstructed by superposing the individual wavefields of primitive structures. Wave-propagation methods generally suffer from two major problems: (i) the high computational cost of superimposing the wavefields of object points at each pixel of a $\mathrm{CGH}^{4}{ }^{4}$ (ii) the occlusion problem, ${ }^{5}$ i.e., for each viewpoint, occluded primitives of a scene should be identified and their contibutions should be removed. If the latter computation is skipped, hidden surfaces become visible and the whole scene becomes transparent in an unnatural fashion. Utilization of graphical processing units (GPUs) and look-up tables are among common practices to mitigate the high computational load.

In ray-tracing-based methods, CGHs are computed by processing 3D scenes with computer graphics methods. In particular, for each viewpoint, computer graphics techniques are utilized to determine perspective views including occlusion effects in a fast and efficient manner. However, in most implementations, the final CGH is formed merely as a collection of the $2 \mathrm{D}$ perspective views, losing the accommodation cue. Holographic stereograms ${ }^{6}$ and integral imaging based displays are well known implementations of this type. In such displays, even though binocular disparity and motion parallax cues may be delivered correctly along with appropriate occlusion effects, all objects appear sharp only when the viewer focuses at a single depth (usually the hologram plane), and apparently out of plane objects appear blurred when focused on. As a result, such implementations are prone to the well known accommodation-convergence conflict typical of stereoscopic displays.

In this work, we present a method to compute a CGH with correct occlusion and accommodation effects in a fast manner. First, given a 3D scene, we utilize a GPU and computer graphics methods to determine perspective

Further author information: (Send correspondence to Seyedmahdi Kazempourradi)

E-mail: mkazempour14@ku.edu.tr

Practical Holography XXXI: Materials and Applications, edited by Hans I. Bjelkhagen,

V. Michael Bove, Jr., Proc. of SPIE Vol. 10127, 101270Q • () 2017 SPIE

CCC code: $0277-786 \mathrm{X} / 17 / \$ 18 \cdot$ doi: $10.1117 / 12.2252374$

Proc. of SPIE Vol. 10127 101270Q-1 
images for a set of viewpoints. In our implementation, we perform the render via the Blender software. Blender is the open source tool for 3D content creation. In addition, we extract and use the depth map (Z-buffer) information that is already computed during rendering calculations. Based on the depth map, we divide the scene into a number of slices, each corresponding to a specific depth. Then, for each slice, we compute an elementary hologram using an FFT based Fresnel transform. We obtain the final CGH for that viewpoint by superposing the elementary holograms of slices. Simulations and experiments verify the proposed method.

\section{COMPUTATION OF COMPUTER-GENERATED HOLOGRAM}

Figure 1 illustrates the $\mathrm{CGH}$ calculation procedure for a single viewpoint. A 3D scene is prepared in Blender software. A virtual camera is placed at the viewpoint position. The field of view (FOV) of the camera is adjusted to match the FOV that can be provided by the SLM. We then extract the perspective intensity image and the normalized 8-bit depth map (Z-buffer), where we benefit a GPU to maximize the computation speed.

Each pixel on the depth map image has a value between 0 and 255, where the black pixels corresponds to the nearest pixels to the camera sensor plane. Since the sensitivity of human visual system to accommodation changes is much lower than 255 levels (3 or 4 bits are usually quite sufficient), the 3D object depth is quantized into several planes. To improve the depth cue effect, we quantize the depth based on linear diopter increments. We then propagate each planar sub-image using an FFT based Fresnel transform to the CGH plane. Afterwards, we sum up the elementary CGHs to obtain the full-complex CGH.

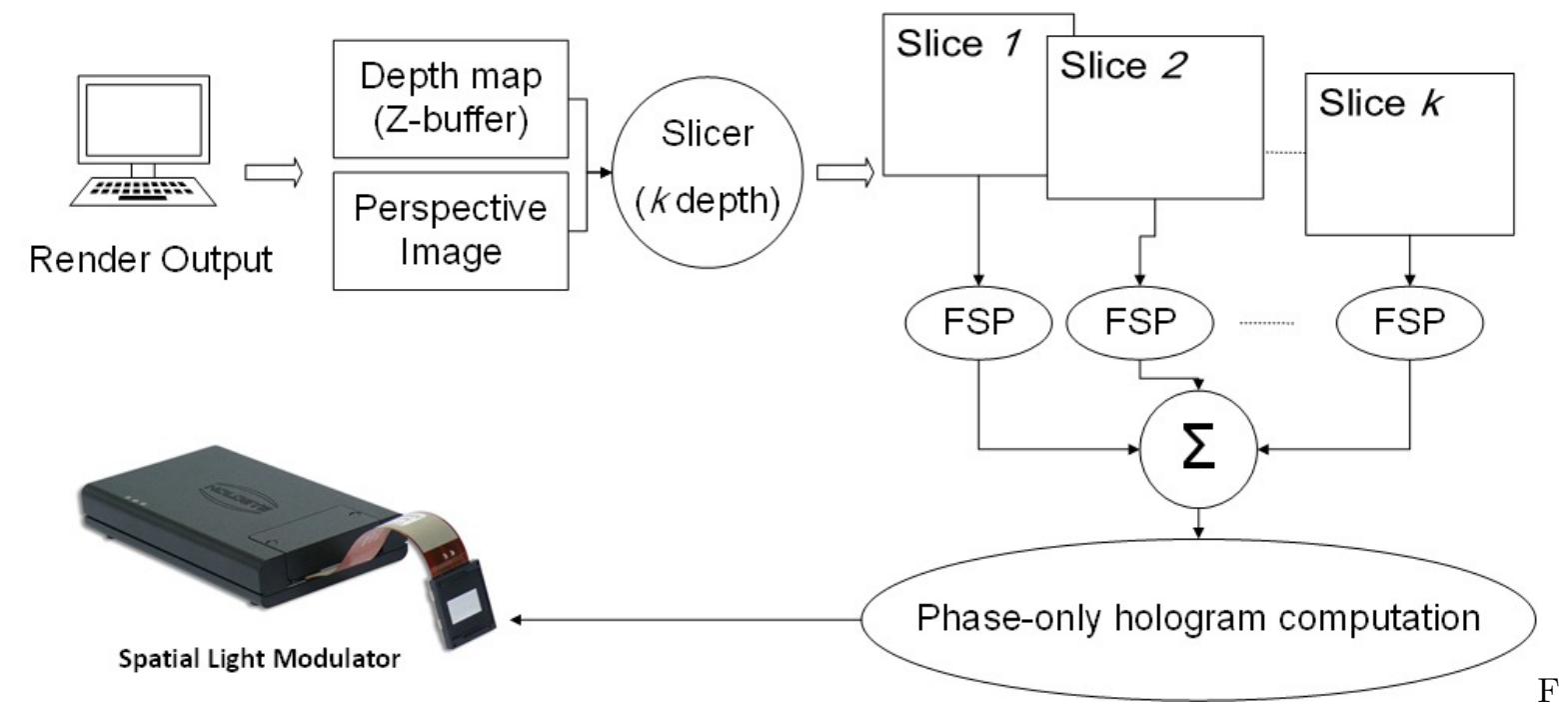

Figure 1. The CGH computation process for a single viewpoint. The render provides a perspective image along with depth map. The object is sliced into several sub-images at different depths. Free-space propagation (FSP) based on an FFT based Fresnel transform is applied on each sub-image to compute elementary holograms. Finally, all elementary holograms are superimposed and the final full-complex CGH is encoded into a phase-only CGH to be displayed on a spatial light modulator (SLM).

Figure 2 illustrates a rendered 3D scene (i.e. the perspective image and depth map), the slices at 8 different planes and the final phase-only CGH.

The final hologram is complex-valued, having both magnitude and phase variation, i.e.,

$$
U_{0}(r)=A_{0}(r) \exp \left(j \Phi_{0}(r)\right)
$$

We use a phase-only CGH to optically reconstruct the holograms. In this respect, the full-complex CGH should be encoded into a phase-only CGH. An option is to simply discard the magnitude information of $U_{0}(r)$. However, this will not result in a high-quality image. Hence, in the last step, we use the well-known iterative Fourier transform algorithm (IFTA) to compute phase-only CGHs. 


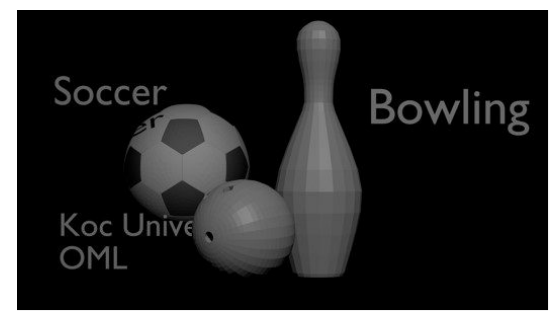

(a) Original Image

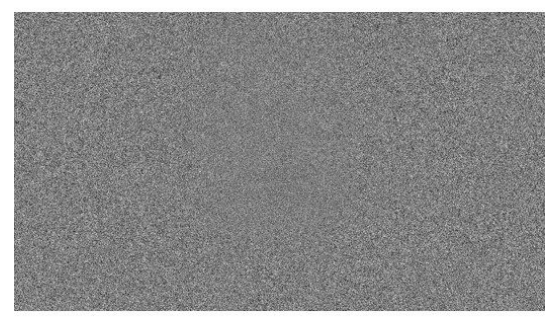

(k) Computed Hologram

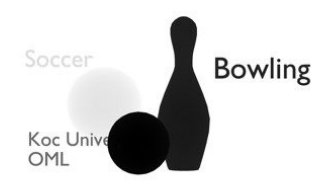

(b) Depth map

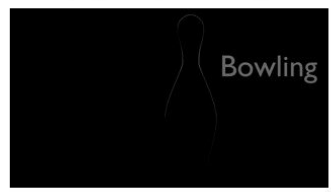

(e) slice 3

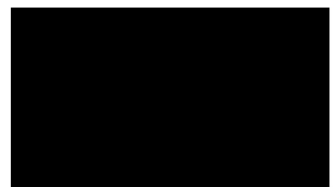

(h) slice 6

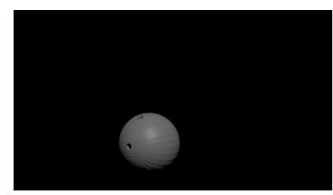

(c) slice 1

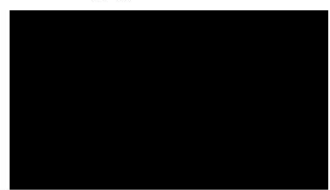

(f) slice 4

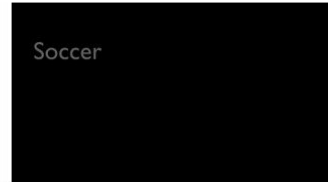

(i) slice 7

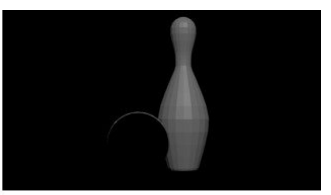

(d) slice 2

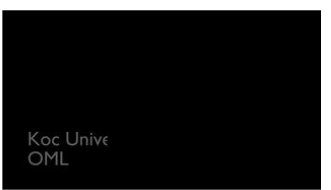

(g) slice 5

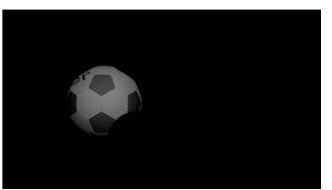

(j) slice 8

Figure 2. (a) Perspective image of a 3D scene, (b) the 8-bit depth map image, (c)-(j) slices of the original image at 8 different planes and $(\mathrm{k})$ the final phase-only $\mathrm{CGH}$.

The IFTA algorithm calculates a phase-only CGH with an engineered spectrum. Specifically, in the spectrum domain the IFTA algorithm

- generates a desired field within $W_{i}$,

- generates zero field within the safety region $W_{s}$,

- distributes the quantization noise to the do not care region $W_{D C}$.

The IFTA algorithm computes a phase only CGH with the following pseudo-code:

Step 0 Generate a random phase-only CGH $\left(\exp \left(j \Phi_{\text {rand }}(r)\right)\right)$

Step 1 Take the Fourier transform and find the output spectrum

Step 2 Leave the spectrum free in $W_{D C}$ region

Step 3 Force the spectrum to zero in $W_{s}$

Step 4 Replace the field in $W_{i}$ with the desired field, after proper normalization such that the replaced and the desired fields have the same energy

Step 5 Take the inverse Fourier transform.

Step 6 Discard magnitude information and keep the phase to find a new phase-only CGH

Step 7 If the maximum iteration number is not reached, go back to Step 1 


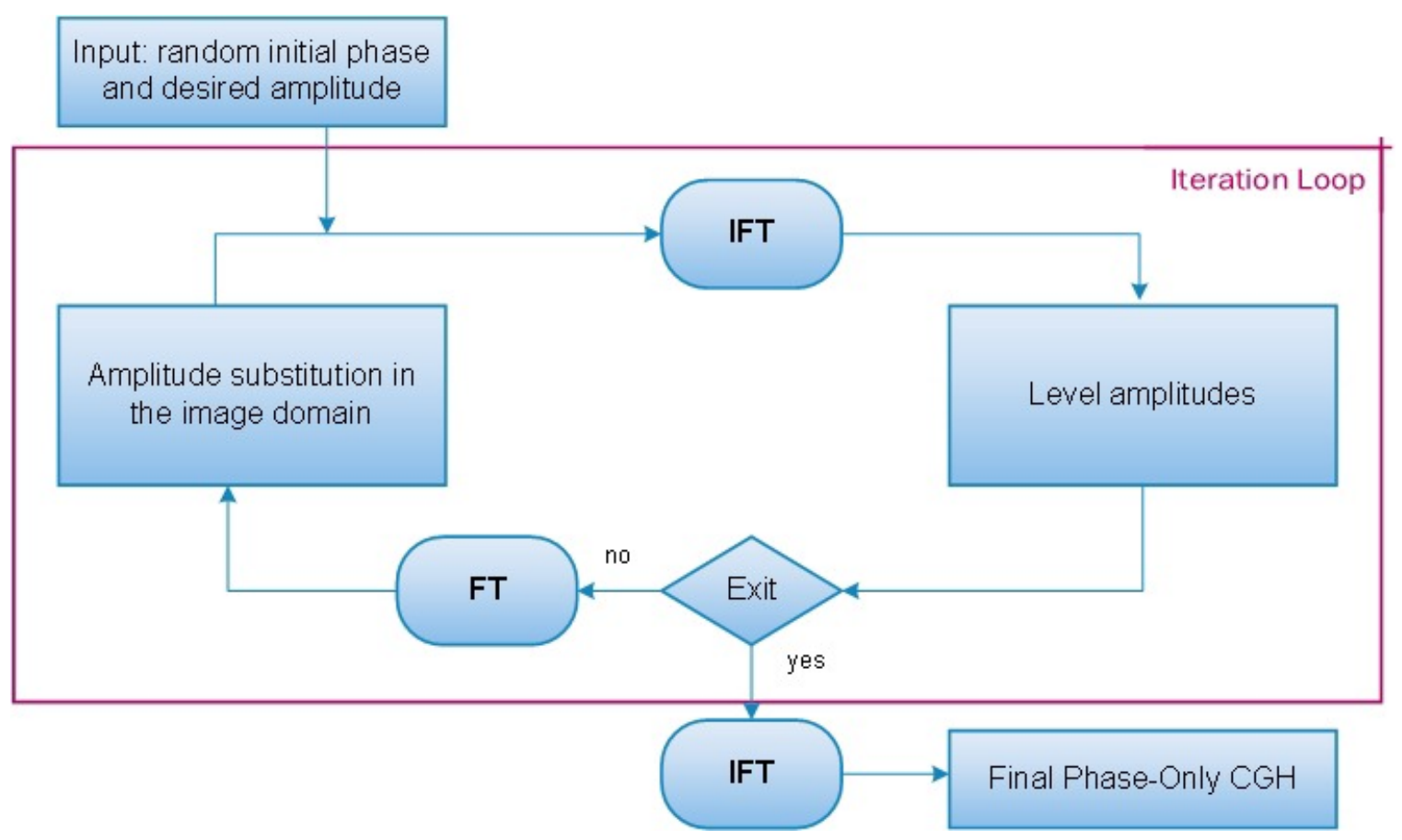

Figure 3. The flowchart of IFTA algorithm. The algorithm performs iterations to compute a phase-only CGH.

\section{EXPERIMENTAL RESULTS}

The proposed method is verified with optical experiments. In the experiments, we use HOLOEYE PLUTO spatial light modulator with a resolution of $1920 \times 1080$ pixels and $8 \mu \mathrm{m}$ pixel pitch. All results are monochrome and we used a He-Ne red laser of wavelength $632.9 \mathrm{~nm}$. The scene in Blender consists of a meshed bowling ball, a meshed bowling bottle together with a "Bowling" text, a "Koc University, OML" text and a soccer ball with a "Soccer" text. These objects are respectively placed at distances of 1, 2, 3 and 4 diopters from the camera sensor plane.

Figure 4 illustrates the experimental images taken from a first viewpoint. As depicted from Fig. 4(a), the "Koc University, OML" text is partially occluded with the bowling ball and bottle objects. Figure 4(b) shows the depth map of this scene. Figures 4(c)-(f) demonstrate the reconstructed images where the focus of camera is changed from the first object to the last object.

Figure 5 shows a set of experimental results from the same scene and a different perspective view. The "Koc University, OML" text is not occluded in this view, as evident in Fig. 5(a). The normalized depth map of the new perspective is shown in Fig. 5(b). The camera focus is changed from the nearest point to the furthest point. The results verify the method and show that the proposed technique is capable to handle both accommodation and occlusion cues.

\section{CONCLUSION}

We propose a method for computing realistic computer-generated holograms (CGHs) of three-dimensional (3D) objects. In this method we utilize computer graphics techniques to handle occlusion, shading and parallax effects in an efficient manner. The depth map (Z-buffer) data of the scene helps us to extract perspective view and the $3 \mathrm{D}$ position of the object. The depth range is divided into several planes. Afterwards, proper FFT of these planar objects is computed in the direction each view point to compute each elementary hologram. Finally, we sum them up to create the final hologram corresponding to the particular viewpoint. The experimental results show that the technique is capable of performing high quality reconstructions in a fast manner. 


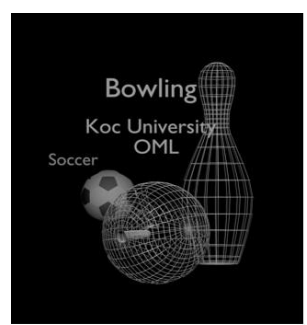

(a)

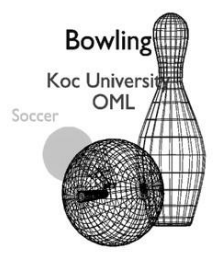

(b)

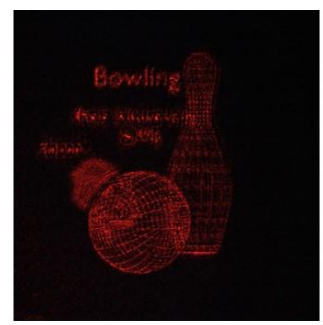

(c)

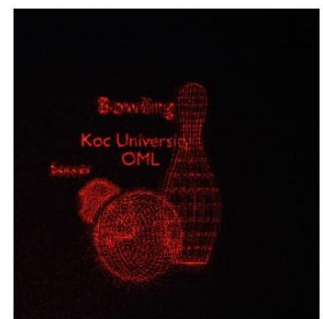

(d)

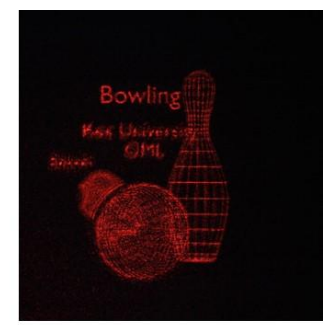

(e)

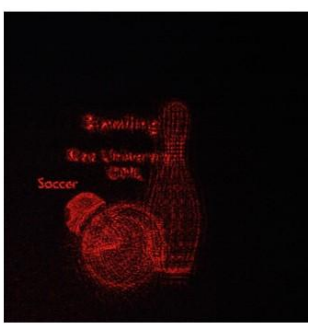

(f)

Figure 4. (a) Original rendered scene, (b) the normalized 8-bit depth map of the scene, (c) reconstructed image with the focus of camera on bowling ball, (d) reconstructed image with the focus of camera on "Koc University" text, (e) reconstructed image with the focus of camera on bowling bottle and "Bowling" text and (f) reconstructed image with the focus of camera on the soccer ball.

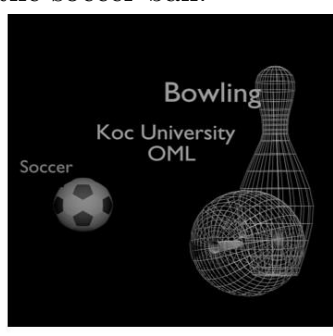

(a)

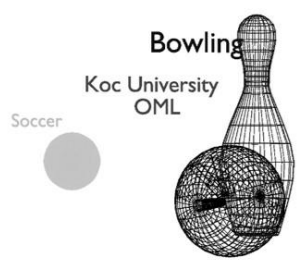

(b)

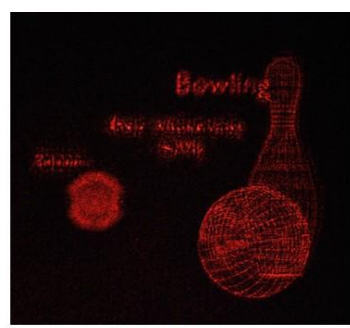

(c)

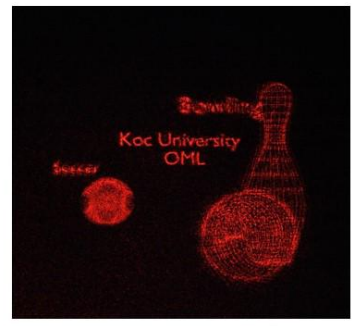

(d)

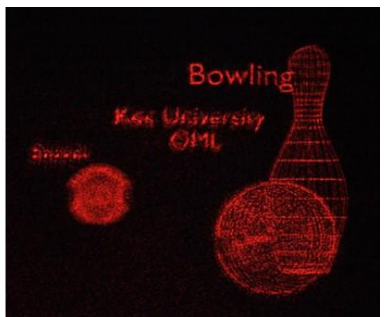

(e)

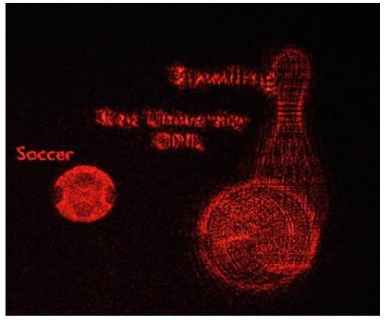

(f)

Figure 5. (a) 3D scene, perspective image (b) the normalized 8-bit depth map of the scene, (c) reconstructed image with the focus of camera on bowling ball, (d) reconstructed image with the focus of camera on "Koc University" text, (e) reconstructed image with the focus of camera on bowling bottle and "Bowling" text and (f) reconstructed image with the focus of camera on the soccer ball.

\section{Acknowledgement}

This work is supported by European Research Council under the Seventh Framework Programme (FP7/20072013) ERC grant agreement no 340200, with acronym Wear3D. 


\section{REFERENCES}

[1] Waters, J. P., "Holographic image synthesis utilizing theoretical methods," Appl. Phys. Lett. 9, 405-406 (1996).

[2] Matsushima, K., "Computer-generated holograms for three-dimensional surface objects with shade and texture," Appl. Opt. 44, 4607-4614 (2005).

[3] Yatagai, T., "Stereoscopic approach to 3-d display using computer-gernerated holograms," Appl. Opt. 15, 2722-2729 (1976).

[4] Pan, Y. C., "Fast cgh computation using s-lut on gpu," Opt. Express 17, 18543-18555 (2009).

[5] Zhang, H., "Full parallax three-dimensional display with occlusion effect using computer-generated hologram," Opt. Eng. 50, 33-42 (2011).

[6] Smithwick, Q. Y. J., "Real-time shader rendering of holographic stereograms," Proc. SPIE 7233, 723302 (2009). 